Pacific Journal of Mathematics

GEOMETRIC STRUCTURE OF ABSOLUTE BASIS SYSTEMS IN 


\section{GEOMETRIC STRUCTURE OF ABSOLUTE BASIS SYSTEMS IN A LINEAR TOPOLOGICAL SPACE}

\section{R. E. Fullerton}

1. Introduction. Let $X$ be a convex linear topological space over the reals. Since most of our results are trivial for finite dimensional spaces, we assume that $X$ is not finite dimensional and that it is complete. An absolute biorthogonal basis system in $X$ consists of an indexed set of elements $\left\{\mathrm{x}_{\alpha}\right\} \subset X, \alpha \in A$ together with a similarly indexed set of linear functionals $\left\{\phi_{\alpha}\right\} \subset X^{*}, \alpha \in A$ such that

(a) $\phi_{\alpha}\left(x_{\beta}\right)=0$ if $\alpha \neq \beta, \phi_{\alpha}\left(x_{\alpha}\right)=1$;

(b) each $x \in X$ is uniquely expressible in the form $x=\sum_{\alpha} \phi_{\alpha}(x) x_{\alpha}$ where $\dot{\phi}_{\alpha}(x)=0$ for all but a countable family of indices $\alpha \in A$ and the sum is independent of the order of summation. We shall investigate the geometric structure of absolute biorthogonal basis systems and shall show that the existence of such a system is equivalent to the existence of a certain type of cone in the space $X$. This work extends further the investigations initiated in the author's note [3].

In particular this theorem closely parallels certain recently announced results of Choquet $[1,2]$ on the existence and uniqueness of representations of elements of a cone in $X$ by means of resultant integrals with respect to Radon measures with supports in the set of extreme points of a base for the cone. In the last section we discuss the theorems of Choquet and indicate the parallelism between his theorems and the theorem discussed above.

2. Basic definitions and notations. We shall define here certain geometric concepts associated with a linear space which we shall need in the discussion to follow. If $X$ is any real linear space and if $x, y \in X, x \neq y$, the segment joining $x$ and $y$ is the set of points $\{a x+(1-a) y\}$, $0 \leqq a \leqq 1$. The ray from $x$ through $y$ is the set $\{a y+(1-a) x\}, a \geqq 0$. A cone $C$ in $X$ with vertex $x$ is a set such that $y \in C, y \neq x$ implies that the ray from $x$ through $y$ lies in $C$. A point $x \in X$ is an extreme point of a set $A \subset X$ if $x \in A$ and if for any segment $\{a u+(1-a) v\}$ with $x=a_{0} u+\left(1-a_{0}\right) v, 0<a_{0}<1$, either $u$ or $v$ is not in $A$. If a ray $r$ is a subset of a convex set $A, r$ is an extreme ray of $A$ if any segment $s=\{a u+(1-a) v\}, \quad 0 \leqq a \leqq 1$ with $x=a_{0} u+\left(1-a_{0}\right) v \in r$, $0<a_{0}<1$ has a subsegment contained in $r$ or either $u$ or $v$ is not in $A$. A cone $C$ is a $C$-cone or minihedral cone if $C$ is a convex cone with its vertex

Received January 18, 1961. This work was done partially during the time the author was a Fellow of the John Simon Guggenheim Memorial Foundation and it was also supported in part by NSF contract NSF G-9423. 
as an extreme point and such that for every pair $x, y \in X$ there exists $z \in X$ such that $(x+C) \cap(y+C)=z+C$. Several elementary properties of $C$-cones should be noted. First, it is evident that any $C$-cone generates the space $X$ in the sense that any element of $X$ is a difference of two elements of $C$. This is true since if $x \in X$, there exists a $z \in X$ with $C \cap(x+C)=z+C$ and for every $v \in C$ there exists a $u \in C$ with $x+u=z+v$ and $x=(z+v)-u$ where $z+v \in C, u \in C$. Secondly, $C$ defines a lattice order on $X$ in which we define $x \geqq y$ if $x-y \in C$ and it is easily verified that if $x, y \in X$, the supremum $x \vee y$ is the element $z \in X$ for which $(x+C) \cap(y+C)=z+C$. Conversely, it is easily seen that the cone of positive elements in a linear lattice is a $C$-cone.

The fundamental cube of Hilbert also occurs extensively in the following discussions. This set can be defined in several equivalent ways. In general, a cube is the topological product of a set of closed unit intervals $P_{\beta} I_{\beta}, \beta \in B$ where the points are all functions $f: B \rightarrow I$ and a neighborhood $N_{f_{0}}$ of $f_{0}$ is determined by any finite set $\beta_{1}, \beta_{2}, \cdots, \beta_{n}$ of indices and open intervals $J_{\beta_{i}}$ of $f_{0}\left(\beta_{i}\right), i=1,2, \cdots, n$ with $f \in N_{f_{0}}$ if $f\left(\beta_{i}\right) \in J_{\beta_{i}}$ for $i=1,2, \cdots, n$. The Hilbert cube $I^{\aleph_{0}}$ can be defined as the topological product of a countably infinite set of unit intervals. The Hilbert cube $I_{1}^{\aleph_{0}}$ can also be equivalently defined as the subset of the Hilbert space $l^{2}$ for which the $i$ th coordinate lies in the closed interval $[0,1 / i]$. It is easily proved that the correspondence $\Phi: I^{\aleph_{0}} \rightarrow I_{1}^{\aleph_{0}}$ defined by associating with $f \in I^{\aleph_{0}}$ the sequence $(f(1), f(2) / 2, f(3) / 3, \cdots)$ in $I_{1}^{\aleph_{0}}$ is a homeomorphism onto $I^{\aleph_{0}}$ which also preserves the linear structure of the cube as a subset of the linear space of real functions over the integers. It is also well known that the Hilbert cube is compact.

A closed half space $H \subset X$ is a set determined by a linear function of $f \in X^{*}$ such that $H=\{x \in X \mid f(x) \geqq 0\}$. We define a hyperplane $L$ to be a set of the form $L=\{x \in X \mid f(x)=0\}$.

3. Absolute basis cones. We shall define the type of cone used to characterize an absolute basis system and develop several of its properties.

Definition. Let $\mathscr{R}=\left\{r_{\alpha}\right\}, \alpha \in A$ be a set of rays in $X$ satisfying the following conditions

(1) $\bigcup_{\alpha \in A} r_{\alpha}$ generates a linear space dense in $X$.

(2) For each $\alpha \in A$ the closed linear subspace $L_{\alpha}$ generated by the $\left\{r_{\beta}\right\}, \beta \neq \alpha$ is a hyperplane.

(3) For each $\alpha \in A, r_{\alpha} \cap L_{\alpha}=\theta$.

(4) If for each $\alpha \in A, H_{\alpha}$ is the closed half space bounded by $L_{\alpha}$ which contains $r_{\alpha}$ and if $K=\bigcap_{\alpha \in A} H_{\alpha}$, then for every $x \in K$, $K \cap(x-K)$ is compact and metrizable.

Then the set $K$ defined in (4) is called an absolute basis cone (a.b.c.).

Theorem 1. Let $K$ be an absolute basis cone. Then $K$ is a C-cone 
with extreme rays $\left\{r_{\alpha}\right\}, \alpha \in A$. Also $K$ is the closed convex hull of the set $\bigcup_{\alpha \in A} r_{\alpha}$.

Proof. The proof will follow as a result of four lemmas. $K$ is evidently a convex cone. For each $\alpha \in A$, let $l_{\alpha}$ be the line containing $r_{\alpha}$ and let $\left\{x_{\alpha}\right\}$ be a bounded set of nonzero elements $x_{\alpha} \in r_{\alpha}, \alpha \in A$.

Lemma 1. $\bigcap_{\beta \neq \alpha_{0}} L_{\beta}=l_{\alpha_{0}}$ for every $\alpha_{0} \in A$.

Proof. Assume that $M=\bigcap_{\beta \neq \alpha_{0}} L_{\beta}$ contains points not on $l_{\alpha_{0}}$. Since $R=\bigcup_{\alpha \in A} r_{\alpha}$ renerates a linear subspace dense in $X, M$ is included in a closed linear manifold generated by a subfamily $\left\{r_{\beta}\right\}, \beta \in \Delta \subset A$. Let $L_{\Delta}$ denote the closed linear subspace determined by the $\left\{r_{\beta}\right\}, B \in \Delta$. Denote by $\mathfrak{M}$ the family of all subsets $\Delta \subset A$ such that $M \subset L_{\Delta}$. $\mathfrak{M}$ can be partially ordered by defining $\Delta_{1}<\Delta_{2}$ if $L_{\Delta_{2}} \subset L_{\Delta_{1}}$. This is a proper partial order since it is evidently transitive and condition (3) insures that the correspondence between $\Delta$ and $L_{\Delta}$ is one-to-one. Let $\mathscr{L}$ be a maximal simply ordered subset of $\mathfrak{M}$. Then the intersection $\Delta_{0}$ of all elements $\Delta \in \mathscr{C}$ is non empty and is a maximal element of $\mathfrak{M}$. Also $M \subset L_{\lrcorner_{0}}$ and no smaller family of rays in $\left\{r_{\alpha}\right\}$ will generate a closed linear subspace containing $M$. However, if $r_{\alpha_{1}} \in L_{\Delta_{0}}, \alpha_{1} \neq \alpha_{0}$ then $r_{\alpha_{1}} \cap L_{\alpha_{1}}=\theta$ and since $M \subset L_{\alpha_{1}}, r_{\alpha_{1}} \cap M=\theta$. Hence $M \subset L_{\lrcorner_{0}} \cap L_{\alpha 1}$ contrary to the maximality of $A_{10}$. This yields a contradiction and shows that $\bigcap_{\beta \neq \alpha_{0}} L_{\beta_{0}}=l_{\alpha}$.

\section{Corollary. $\bigcap_{\alpha \in A} L_{\alpha}=\theta$.}

Lemma 2. Let $K^{\prime}$ be the closure of the convex set determined by the family $\left\{r_{\alpha}\right\}, \alpha \in A$ where $\left\{r_{\alpha}\right\}$ satisfies the conditions of the definition. Then $K=K^{\prime}$.

Proof. Obviously $K^{\prime} \subset K$. Assume that there exists a point $x_{0} \in K \backslash K^{\prime}$. This implies that $x_{0}$ is not the limit point of any net $\left\{y_{\gamma}\right\} \subset X, \gamma \in \Gamma$, of elements of the form $y_{\gamma}=\sum_{i=1}^{n_{\gamma}} a_{\alpha_{i}}^{\gamma} x_{\alpha_{i}}$ with $a_{x_{i}}^{\gamma}>0, i=1,2, \cdots, n_{\gamma}$, since all such elements are in $K^{\prime}$. For any $x \in X$ it is possible to define a unique set of real coordinates $a_{\alpha}(x)$ indexed by $A$ as follows. Let $a_{\alpha}(x)=\sup \left\{a \mid x \in a x_{\alpha}+H_{\alpha}\right\}$. Then $\left|a_{\alpha}(x)\right|<\infty$ and $x \in K$ evidently implies $a_{\alpha}(x) \geqq 0$ for all $\alpha \in A$. Now let $\Gamma$ denote the family of all finite subsets of $A$ ordered by inclusion. $\Gamma$ is a directed set. For $\gamma \in \Gamma$, let $y_{\gamma}=\sum_{\alpha \in \gamma} a_{\alpha}\left(x_{0}\right) x_{\alpha}$. Then $\left\{y_{\gamma}\right\}$ is a net with its elements all in $K^{\prime}$. Also $y_{\gamma} \in K \cap\left(x_{0}-K\right)$ for each $\gamma \in \Gamma$. By the compactness of $K \cap(x-K)$, the net $\left\{\mathrm{y}_{\gamma}\right\}$ contains a convergent subnet $\left\{y_{\gamma_{\delta}}\right\}$ converging to an element $y_{0} \in K \cap\left(x_{0}-K\right)$. By using the Hahn Banach theorem, it can be seen that convergence of a net of elements in $X$ implies 
coordinatewise convergence. Hence $\lim a_{\alpha}\left(y_{\gamma_{\delta}}\right)=a_{\alpha}\left(y_{0}\right)$ for each $\alpha \in A$. However $a_{\alpha}\left(y_{\gamma}\right)$ is either $a_{\alpha}\left(x_{0}\right)$ or 0 by the definition of $y_{\gamma}$. Since $\left\{\gamma_{\delta}\right\}$ is cofinal with $I^{\prime}$, eventually $a_{\omega}\left(y_{\gamma_{\delta}}\right)=a_{\alpha}\left(x_{0}\right)$ for each $\alpha \in A$. Thus $a_{\alpha}\left(y_{0}\right)=a_{\alpha}\left(x_{0}\right)$ for each $\alpha \in A$. However, this implies that $y_{0}=x_{0}$ since $x_{0}-y_{0} \in \bigcap_{\alpha \in A} \Gamma_{\alpha}=\theta$. Thus if $x_{0} \in K$ it is the limit of a net of elements in $K^{\prime}$ and since $K^{\prime}$ is closed, $K=K^{\prime}$.

Note. It would be sufficient for this lemma to assume that $K^{\prime} \cap(x-K)$ is compact instead of condition (4). Whether it is sufficient to replace (4) by the metrizability and compactness of $K^{\prime} \cap\left(x-K^{\prime}\right)$ is not known to the author at present.

Lemma 3. Let $\Delta \subset A, \Gamma \subset A$ be two complementary sets of indices, i.e. $\quad A=\Delta \cup \Gamma, \Delta \cap \Gamma=\phi$. Let $L_{\Delta}, L_{\Gamma}$ be respectively the closed linear subspace generated by $\left\{r_{\hat{o}}\right\}, \delta \in \Delta ;\left\{r_{\gamma}\right\}, \gamma \in \Gamma$ respectively. Then $L_{\Delta} \cap L_{\Gamma}=\theta$.

Proof. Assume that $y \in L_{\Delta} \cap L_{\gamma}$. Evidently $L_{\Delta} \subset L_{\gamma}$ for each $\gamma \in \Gamma$ and $L_{\Gamma} \subset L_{\delta}$ for each $\delta \in \Delta$. Since $y \in L_{\Delta} \cap L_{\Gamma} \subset \bigcap_{\alpha \in A} L_{\alpha}=\theta, y=\theta$.

Lemma 4. If $x, y \in X$ and $\Delta, \Gamma$ are complementary subsets of $A$ then $\left(x+L_{\Delta}\right) \cap\left(y+L_{\Gamma}\right)$ contains exactly one point.

Proof. By a translation if necessary, we may assume that $y=\theta$. We show first that $X=L_{r}+L_{\Delta}$. Since for any $x \in X$, there exist $x_{1}, x_{2} \in K$ with $x=x_{1}-x_{2}$, it is sufficient to show that for $x \in K, x=u+v$, $u \in L_{\Delta}, v \in L_{l}$. Let $\left\{a_{\alpha}(x)\right\}$ be the coordinates of $x$ as described in Lemma 2 and let $\left\{y_{\lambda}\right\}$ be the net defined in Lemma 2 of finite linear combinations of $\left\{x_{\alpha}\right\}$ with coefficients $a_{\alpha}(x)$ which converges to $x$.

If

$$
\begin{aligned}
& y_{\lambda}=\sum_{i=1}^{n_{\lambda}} a_{\alpha_{i}}(x) x_{\alpha_{i}}, \\
& u_{\lambda}=\sum_{i=1}^{n} a_{\alpha_{i}} \hat{\varkappa}_{\alpha_{i}}, \\
& v_{\lambda}=\sum_{i=1}^{N_{\lambda}} c_{\alpha_{i}} x_{\alpha_{i}}
\end{aligned}
$$

where $a_{\alpha_{i}}=a_{\alpha_{i}}(x)$ if $\alpha_{i} \in \Delta$ and $a_{\alpha_{i}}=0$ otherwise and let $c_{\alpha_{i}}=a_{\alpha_{i}}(x)$ if $\alpha_{i} \in \Gamma$ and 0 otherwise. Then the nets $\left\{u_{\lambda}\right\},\left\{v_{\lambda}\right\}$ lie in $K \cap(x-K)$ and hence have convergent subnets. However, as in Lemma 2 , these convergent subnets converge coordinatewise and hence if $\lim _{\eta} u_{\lambda_{\eta}}=u_{0}$, $\lim _{\mu} v_{\lambda_{\mu}}=v_{0}$ then $u_{0}$ has as its coordinates $a_{\alpha}(x)$ if $\alpha \in \Delta$ and 0 if $\alpha \in I^{\prime}$; $v_{0}$ has as its coordinates $a_{\alpha}(x)$ if $\alpha \in \Gamma$ and 0 if $\alpha \in \Delta$. However, it is easily seen that each coordinate of the sum of two elements is the sum 
of the coordinates and hence $x=u_{0}+v_{0}$. Thus $X=L_{\Delta}+L_{l}$. Moreover, Lemma 3 shows that this decomposition is unique.

To finish the proof, consider $L_{\Gamma} \cap\left(x+L_{\Delta}\right)$ for any $x \in X$. Since $x=u+v$ for $u \in L_{I}, v \in L_{\lrcorner}, x-u=v$ and since $x-u \in x+L_{\Delta}, v \in L_{\Gamma}$, $v \in L_{i} \cap\left(x+L_{\Delta}\right) \neq \phi$. By the uniqueness of $u$ and $v, L_{\Gamma} \cap\left(x+L_{\Delta}\right)=v$ and the intersection consists of exactly one point.

Proof of the theorem. It is sufficient to show that for any $x \in X$, there exists $z \in X$ such that $K \cap(x+K)=z+K$. We construct $z$ as follows. For each $\alpha \in A$, let $\alpha_{\alpha}(x)$ be the $\alpha$ coordinate of $x$. If $a_{\alpha}(x) \leqq 0$ let $\alpha \in \Delta$ and if $a_{\alpha}(x)>0$, let $\alpha \in \Gamma$. Evidently $\Delta \cup \Gamma=A, \Delta \cup \Gamma=\phi$. Define $L_{\Delta}, L_{j}$ as above. Let $z=L_{r} \cap\left(x+L_{\Delta}\right)$. It must be shown that $K \cap(x+K)=z+K$. By construction $a_{\alpha}(z) \geqq 0$ for all $\alpha \in A$. Hence $z \in K$. Also, since $x+K$ is the set of all elements $y \in X$ for which $a_{\alpha}(y) \geqq a_{\alpha}(x), \alpha \in A, z \in x+K$. Hence $z+K \subset K \cap(x+K)$.

To show the reverse inclusion, assume that $y \in K \cap(x+K)$ but $y \notin z+K$. Since $y \in K \cap(x+K), y \in H_{\alpha}$ and $y \in x \quad-H_{\alpha}$ for all $\alpha \in A$. Since $y \notin z+K, \quad y \notin z+H_{\alpha}$ for some $\alpha_{0} \in A$. This implies that $a_{\alpha_{0}}(y)<\alpha_{\alpha_{0}}(z)$. If $\alpha_{0} \in \Gamma, z+H_{\alpha_{0}}=x+H_{\alpha_{0}}$ and $y \notin x+H_{\alpha_{0}}$. Hence $y \notin x+K$. If $\alpha_{0} \in \Delta, y \notin z+H_{\alpha_{0}}=H_{\alpha_{0}}$ and $y \notin K$. Thus in either case $y \notin K \cap(x+K)$ contrary to assumption. Hence $K \cap(x+K)=z+K$ and $K$ is a $C$-cone.

To prove that each $r_{\alpha}$ is an extreme ray, if for $\alpha_{0} \in A, r_{\alpha_{0}}$ were not an extreme ray, then $\bigcap_{\beta \neq \alpha_{0}} L_{\beta}$ includes a two dimensional subspace, contrary to Lemma 1.

TheOREM 2. If $K$ is an absolute basis cone in $X$, then for every $x \in K$, the set $P_{x}=K \cap(x-K)$ is linearly homeomorphic to a finite dimensional cube or to the fundamental cube of Hilbert.

Proof. For each $\alpha \in A$, let $I_{x}^{\prime}$ be the real interval $0 \leqq t \leqq a_{\alpha}(x)$ where $a_{\alpha}(x)$ is the $\alpha$ coordinate of $x$ as defined in Theorem 1, Lemma 2. Let $A_{x} \subset A$ be the set of indices in $A$ for which $a_{x}(x) \neq 0$. Then every $y \in P_{x}$ has coordinates $\left\{a_{\alpha}(y)\right\}, \alpha \in A_{x}$ where $0 \leqq a_{\alpha}(y) \leqq a_{\alpha}(x), \alpha \in A_{x}$. Thus there exists a one-to-one correspondence between $P_{x}$ and a subset of the cartesian product $\mathscr{P}_{\alpha} I_{\alpha}^{*}, \alpha \in A_{x}$. The correspondence is one-to- $\delta \mathrm{e}$ since if $y_{1} \neq y_{2}$ but $a_{\alpha}\left(y_{1}\right)=a_{x}\left(y_{2}\right)$ for $\alpha \in A_{x}$, then $y_{1}-y_{2} \in \bigcap_{\alpha \in A} L_{\alpha}=\theta$, contrary to assumption. Denote the correspondence by $\Phi: P_{x} \rightarrow \mathscr{P}_{\alpha} I_{\alpha}^{x}$, $\alpha \in A_{x}$.

To show that $\Phi$ is a homeomorphism it need only be shown that $\Phi$ is continuous since $P_{x}$ is compact by hypothesis. Let $\left\{y_{\lambda}\right\}, \lambda \in A$ be a net of elements of $P_{x}$ converging to $y_{0} \in P_{x}$. Let $\left\{a_{\alpha}\left(y_{\lambda}\right)\right\},\left\{a_{\alpha}\left(y_{0}\right)\right\}, \alpha \in A_{x}$, be respectively the coordinates of $y_{\lambda}, y_{0}$ for each $\lambda \in \Lambda$. If $\left\{\Phi\left(y_{\lambda}\right)\right\}$ does 
not converge to $\left\{\Phi\left(y_{0}\right)\right\}$ in $\mathscr{P}_{\alpha} I_{\alpha}^{x}$, this implies that for some $\alpha_{0} \in A_{x}$, $\left\{a_{\alpha_{0}}\left(y_{\lambda}\right)\right\}$ does not converge to $a_{\alpha_{0}}\left(y_{0}\right)$. By definition of the coordinates, this implies that there exists a translate $u+L_{\alpha_{0}}$ of the closed hyperplane $L_{\alpha_{0}}$ which separates $y_{0}$ from a subnet $\left\{y_{\lambda_{y}}\right\}$ of $\left\{y_{\lambda}\right\}$. Hence there exists a neighborhood $U$ of $y_{0}$ which lies completely on one side of $u+L_{\alpha_{0}}$ and hence contains none of the points $\left\{y_{\lambda_{2}}\right\}$. However, this contradicts the convergence of $\left\{y_{\lambda}\right\}$ to $y_{0}$. Thus $\Phi$ is continuous and hence, a homeomorphism.

It must next be shown that $\Phi$ maps $P_{x}$ onto $\mathscr{P}_{\alpha} I_{\alpha}^{x}$. Let $\left\{a_{\alpha}\right\}$ be any element of $\mathscr{P}_{\alpha} I_{\alpha}^{x}$. This implies that $0 \leqq a_{\alpha} \leqq a_{\alpha}(x), \alpha \in A_{x}$. Let $\sigma=\left(\alpha_{1}, \alpha_{2}, \cdots, \alpha_{k}\right)$ denote a finite set of indices in $A_{x}$. Define $\left\{\alpha_{\alpha}^{\sigma}\right\} \in \mathscr{P}_{\alpha} I_{\alpha}^{x}$ as follows. $a_{\alpha_{i}}^{\sigma}=a_{\alpha_{i}}, \alpha_{i} \in \sigma$ and $a_{\alpha}^{\sigma}=0, \alpha \notin \sigma$. Then for every $\sigma$, the element $a_{\alpha}^{\sigma}$ corresponds to an element $y_{\sigma} \in P_{x}, y_{\sigma}=\sum_{i=1}^{k} a_{\alpha_{i}}(x) x_{\alpha_{i}}$. The set $\{\sigma\}$ of all such finite subsets forms a directed set under inclusion and hence the set $\left\{y_{\sigma}\right\}, \sigma \in\{\sigma\}$ is a net of elements in $P_{x}$ with $\Phi\left(z_{\sigma}\right)-\left\{a_{\sigma}^{\alpha}\right\}$ for each $\sigma$. However, the compactness of $P_{x}$ implies that $\left\{y_{\sigma}\right\}$ contains a subnet converging to an element $y_{0} \in P_{x}$. By the construction of the net $\left\{y_{\sigma}\right\}$ this implies that $a_{\alpha}\left(y_{0}\right)=a_{\alpha}$ for every $\alpha \in A_{x}$. Hence $\Phi\left(y_{0}\right)=\left\{a_{\alpha}\right\}$. Thus $\Phi$ maps $P_{x}$ onto $\mathscr{P}_{\alpha} I_{\alpha}^{x}$. Hence $P_{x}$ and $\mathscr{P}_{\alpha} I_{\alpha}^{x}$ are homeomorphic. If we now define $b_{\alpha}(y)=a_{\alpha}(y) / a_{\alpha}(x)$ for $\alpha \in A_{x}$, then the mapping $\Phi^{\prime}(y)=\left\{b_{\alpha}(y)\right\}$ is a homeomorphism of $P_{x}$ onto the cube $\mathscr{P}_{\alpha} I_{\alpha}$ where $I_{\alpha}=[0,1]$ for each $\alpha \in A_{x}$. It is easily seen that $\Phi^{\prime}$ preserves the linear structure of $P_{x}$ and hence that $\Phi^{\prime}$ is a linear homeomorphism of $P_{x}$ onto $\mathscr{P}_{\alpha} I_{\alpha}$.

To complete the proof, it need only be shown that $A_{x}$ is a finite or countable set of indices in $A$. This follows from the metrizability of $P_{x}$. Since $P_{x}$ is metrizable, $\mathscr{P}_{\alpha} I_{\alpha}, \alpha \in A_{x}$ is also. However, if $A_{x}$ is uncountable, it is well known that $\mathscr{P}_{\alpha} I_{\alpha}$ does not satisfy the first countability axiom and is hence not metrizable. This completes the proof of the theorem.

THEOREM 3. A necessary and sufficient condition that $X$ possess an absolute biorthogonal basis system is that $X$ contain an absolute basis cone.

Proof. We first prove the necessity. Assume that $X$ has an absolute basis system $\left\{x_{\alpha}, \phi_{\alpha}\right\}, \alpha \in A$. For each $\alpha \in A$, let $r_{\alpha}$ be the ray $\left\{k x_{\alpha}\right\}$, $k \geqq 0$. Let $K$ be the closure of the convex set determined by the $\left\{r_{\alpha}\right\}$. We prove that $K$ is an a.b.c.

Evidently $K$ is a cone and since the $\left\{x_{\alpha}\right\}$ are points of a basis system, $\mathrm{U}_{\alpha \in A} r_{\alpha}$ generates a linear space dense in $K$. Hence Condition (1) is satisfied.

To prove (2), note that for every $a \in A, L_{\alpha}$ is the null space of a continuous linear functional $\phi_{\alpha}$ and is hence a closed hyperplane. Con- 
dition (3) follows since $\phi_{\alpha}\left(x_{\alpha}\right)=1, \phi_{\alpha}\left(x_{\beta}\right)=0, \beta \neq \alpha$ and hence $r_{\alpha} \cap L_{\alpha}=\theta$.

To prove that Condition (4) holds, we shall show that for each $x \in K$, the set $P_{x}=K \cap(x-K)$ is homeomorphic to a cube of finite or countable dimension and hence compact and metrizable. Let $x \in K$. Then $x=\sum_{i=1}^{\infty} \phi_{\alpha_{i}}(x) x_{\alpha_{i}}$ or $x=\sum_{i=1}^{n} \phi_{\alpha_{i}}(x) x_{\alpha_{i}}$ where $\phi_{\alpha_{i}}(x)>0$ for all $i$ in the sum. Assume that $x$ has a countably infinite expansion, since the proof for the finite case follows from the same type of arguments. For brevity we shall write $x=\sum_{i=1}^{\infty} a_{i} x_{i}$. To each $y \in P_{x}$ let $y=\sum_{i=1}^{\infty} b_{i} x_{i}$. The sequence $\left\{b_{i}\right\}$ is uniquely determined and it is easily seen that $0 \leqq b_{i} \leqq a_{i}, i=1,2, \cdots$. Let $\Phi: P_{x} \rightarrow I^{\aleph_{0}}$ be defined by

$$
\Phi(y)=\left(\frac{b_{1}}{a_{1}}, \frac{b_{2}}{a_{2}}, \frac{b_{3}}{a_{3}}, \cdots\right) \text {. }
$$

To show that $\Phi$ is one-to-one and onto, we must show that every element of $I^{\aleph_{0}}$ is the image of exactly one element of $P_{x}$. If $\left(\zeta_{1}, \zeta_{2}, \cdots\right) \in I^{\aleph_{0}}$, let $b_{i}=a_{i} \zeta_{i}, \quad i=1,2,3, \cdots$ and consider the sum $\sum_{i=1}^{\infty} b_{i} x_{i}$. If this sum converges, it represents an element of $P_{x}$ which maps onto the given element of $I^{\aleph_{0}}$ under $\Phi$.

Let $P_{x}^{m, n}$ be the parallelotope with one vertex at $\theta$ and with edges adjacent to $\theta$ ending at $\left\{a_{i} x_{i}\right\}, i=n, n+1, \cdots, m$. By elementary methods, it can be shown that $P_{x}^{m, n}$ is the convex set determined by all points of the form $\left\{\sum_{j=1}^{k} a_{i_{j}} x_{i_{j}}\right\}$ where the sets $\left\{i_{1}, i_{2}, \cdots, i_{k}\right\}$ run over all finite subsets of the numbers $(n, n+1, \cdots, m)$. However, by the unconditional convergence of $\sum_{i=1}^{\infty} a_{i} x_{i}$, for any neighborhood $U$ of $\theta$ there exists an integer $n_{U}$ such that $\sum_{j=1}^{k} a_{i_{j}} x_{i_{i}} \in U$ for all sets $\left(i_{1}, i_{2}, \cdots, i_{k}\right)$ where $i_{j}>n_{U}, i=1,2, \cdots, k$. Since $U$ is convex, $P_{x}^{m, n} \subset U$ for $m, n>n_{U}$. Hence, since $0 \leqq b_{i} \leqq a_{i}$ for each $i$, we have $P_{y}^{m, n} \subset P_{a}^{m, n}$ for every $m, n$. In particular, $P_{y}^{m, n} \subset U$ if $P_{x}^{m, n} \subset U$. Thus the sequence $\left\{\sum_{i-n}^{m} b_{i} x_{i}\right\}$ converges to $\theta$ if $m, n \rightarrow \infty$. Hence $\Phi$ is a one-to-one transformation of $P_{x}$ onto $I^{\aleph_{0}}$.

Evidently $\Phi$ preserves the linear structure of $P_{x}$. Since $I^{\aleph_{0}}$ is metrizable and compact, to show that $\Phi$ is a homeomorphism we need only prove that $\Phi^{-1}$ is continuous. Let $v \in I^{\boldsymbol{N}_{0}}$ have coordinates $\left(\zeta_{1}, \zeta_{2}, \cdots\right)$, $0 \leqq \zeta_{i} \leqq 1$. Then $\Phi^{-1}(v)=y=\sum_{i=1}^{\infty} \zeta_{i} a_{i} x_{i}=\sum_{i=1}^{\infty} b_{i} x_{i}$. Let $U$ be a neighborhood of $\theta$ in $X \cap P_{x}$. By the preceding paragraph, there exists an integer $n_{U}$ such that if $m>n_{U}, \sum_{i=m}^{\infty} c_{i} x_{i} \in \frac{1}{3} U$ for all choice of $\left\{c_{i}\right\}$ with $0 \leqq c_{i} \leqq a_{i}$. Evidently the mapping $\sum_{i=1}^{n_{U}} c_{i} x_{i}$ is continuous from the finite parallelotope $I_{1} \times I_{2} \times \cdots \times I_{n_{U}} ; I_{j}=[0,1], j=1,2, \cdots, n_{U}$, into $X$. Hence there exists a $\delta>0$ such that $\left(\sum_{i=1}^{n_{I}} c_{i} x_{i}-\sum_{i=1}^{n_{U}} b_{i} x_{i}\right) \in \frac{1}{3} U$ for all finite sequences $\left(c_{1}, c_{2}, \cdots, c_{n_{U}}\right)$ for which $\left|b_{\imath}-c_{i}\right|<\delta, i=1,2, \cdots, n_{U}$. Thus we have

$$
\sum_{\imath=1}^{\infty} c_{i} x_{\imath}-y=\left(\sum_{\imath=1}^{n_{I}} c_{i} x_{i}-\sum_{\imath=1}^{n_{U}} b_{i} x_{i}\right)+\sum_{i=n_{U}}^{\infty} c_{i} x_{i}-\sum_{\imath=n_{U}}^{\infty} b_{i} x_{\imath} \in U
$$


for all sequences $c_{i}$ such that $\left|c_{i}-b_{i}\right|<\varepsilon, i=1,2, \cdots, n_{U}$. If $V$ is a neighborhood in $I^{\aleph_{0}}$ for which $\left|\xi_{i}-\left(b_{i} / a_{i}\right)\right|<\varepsilon / a, \quad a=\max a_{i}$ for $i=1,2, \cdots, n_{U}$ and unrestricted in the other coordinates, then $\Phi^{-1}(V) \subset U$ and $\Phi^{-1}$ is continuous. Hence $\Phi$ is a homeomorphism and $P_{x}$ is compact and metrizable. This shows that $K$ is an absolute basis cone.

Conversely, assume that $X$ contains an absolute basis cone $K$. By Theorem $1 . K$ is a $C$-cone and hence the linear space generated by $K$ is $X$. Hence every element $x \in X$ is uniquely expressible as the difference of two elements of $K$, i.e., if we consider $K$ as defining a lattice order on $X$, then $x=(x \vee \theta)-[-(x \wedge \theta)]$. Hence to show that the set $\left\{x_{a}\right\}$ which defines $K$ constitutes a set of absolute basis elements, it is sufficient to prove that every element of $K$ can be uniquely represented as a countable linear combination of elements of this set. Let $x \in K$ and $P_{x}=K \cap(x-K)$. Let $\left\{a_{x}(x)\right\}$ be the coordinates of $x$ as defined in Theorem 1 and consider the formal sum $\sum_{\alpha \in A_{x}} a_{\alpha}(x) x_{\alpha}$ where as in Theorem 2, $A_{x} \subset A$ is the set of elements of $A$ for which $a_{\alpha}(x)>0$. By Theorem 2, $P_{x}$ is linearly homeomorphic to a finite cube or to the Hilbert cube $I^{\aleph_{0}}$. Let $I^{\aleph_{0}}$ be represented as the set $\{y=$ $\left.\left(\zeta_{1}, \zeta_{2}, \cdots\right) \in l^{2} \mid 0 \leqq \zeta_{\nu} \leqq 1 / i, i=1,2, \cdots\right\}$. Then, by the correspondence set up in Theorem $2, A_{x}$ is finite or countably infinite and if $x=$ $\sum_{\imath=1}^{\infty} a_{\alpha_{i}}(x) x_{\alpha_{i}}, a_{\alpha_{i}}(x) x_{\alpha_{i}}$ corresponds to the point $y_{i}=(0,0, \cdots, 0,1 / i, 0, \cdots)$ where $1 / i$ occurs in the $i$ th place. Thus $\sum_{\alpha \in_{A_{x}}} a_{\alpha}(x) x_{\alpha}$ corresponds to the sum $\sum_{i=1}^{\infty} y_{i}=\left(1, \frac{1}{2}, \frac{1}{3}, \cdots, 1 / i, \cdots\right) \in l^{2}$ under the linear homeomorphism. Since the series $\sum_{i=1}^{\infty} y_{i}$ converges in $l^{2}$, the series $\sum_{a \in A_{x}} a_{\alpha}(x) x_{\alpha}$ converges in $X$ by the definition of the $\left\{a_{x}(x)\right\}$, convergence implies convergence to $x$. For $A_{x}$ a finite set the modifications needed in the above argument are obvious. The expansion is evidently unique and hence the set $\left\{x_{\alpha}\right\}$ is a set of elements of an absolute biorthogonal basis system in $X$.

COROLlary. A necessary and sufficient condition that a locally convex linear topological space $X$ should possess an absolute biorthogonal basis system is that there exist a lattice ordering on $X$ which is compatible with the topological and linear structure of $X$ and for which the following additional conditions are satisfied.

(1) There exists a family $\left\{x_{\alpha}\right\}, \alpha \in A$ of elements in $X$ which are positive, uhich generate a linear space dense in $X$ and such that for every $\alpha \in A$, the set $\left\{y \in X \mid \theta \leqq y \leqq x_{\alpha}\right\}$ is the line segment joining $\theta$ and $x_{\alpha}$.

(2) For every $\alpha \in A$, the closed linear subspace $L_{\alpha}$ generated by the $\left\{x_{\beta}\right\}, \beta \neq \alpha$ is a hyperplane not containing $x_{\alpha}$.

(3) Every ordered interval $I(x, y)=\{z \in X \mid x \leqq z \leqq y\}$ is compact and metrizable.

Proof. This is simply Theorem 3 restated in terms of an order relation. 
It is to be noted that in many of the common function spaces and sequence spaces, the partial order defined by an absolute basis cone does not necessarily coincide with the natural order defined in terms of function values. For example, in the space $c$ of convergent real sequences, the standard basis system has for its set of basis vectors, the set $x_{0}=(1,1,1, \cdots), x_{1}=(1,0,0, \cdots), x_{2}=(0,1,0,0, \cdots), \cdots$. The element $x_{0}-\sum_{i=1}^{\infty} a_{i} x_{i}$ is positive in the natural order if for each $i, 0 \leqq a_{i} \leqq 1$. However, this element is not positive in the partial order defined by the basis cone since this implies that all coefficients of the basis vectors must be nonnegative. In the space $c$ it can be seen that the basis cone can never coincide with the natural positive cone for any basis since the natural positive cone has a nonvoid interior while a basis cone, being determined as the closure of the convex set determined by a family of extreme rays, always has a void interior for $X$ not of finite dimension. By a theorem of Kelley and Vaught [5], it can be seen by similar reasoning that in a real Banach algebra, a basis cone can never coincide with the cone of elements which are squares.

4. Relation to the theorems of Choquet. Choquet $[1,2]$ has announced several theorems concerning the representation of points of a cone $K$ in a locally convex space $X$ in terms of resultant integrals. If $K$ is a cone in $X$, a base $B$ for $K$ is the intersection of $K$ with hyperplane $M$ translated so that it does not contain the origin but which intersects every ray of $K$. Let $K$ be a $C$-cone and assume that $K$ has a base $B$ which is compact and metrizable. Let $E$ be the set of extreme points of $B$. Then Choquet's theorems state that there exists a one-to-one correspondence between points of $K$ and Radon measures $\mu$ whose support is contained in $E$ such that each $x \in K$ is uniquely representable in the form $x=\int_{E} y d \mu$ where $y$ is the function $f(y)=y, y \in E$.

Theorem 3 can be rephrased to yield a similar theorem. This reads as follows.

Theorem 3'. Let $K$ be an absolute basis cone. Let $R \subset K$ be a set of elements $\left\{x_{\alpha}\right\}, \alpha \in A, x_{\alpha} \in r_{\alpha}, \alpha \in A, x_{\alpha} \neq \theta$ for all $\alpha$. Then there exists a one-to-one correspondence between points of $K$ and finite countably additive atomic measures $\nu$ on $R$ whose support is a countable subset of $R$ such that for each $x \in R$, the correspondence between $x$ and $\nu$ is given by $x=\int_{R} y d \nu$.

It can be seen that if $R$ is countable, the conclusion of the theorem would read that the stated correspondence would be between $R$ and all Radon measures on $R$ and would be essentially the same as the conclusion 
of the Choquet theorem.

This theorem would seem to imply that if $K$ is a $C$-cone satisfying Conditions (1), (2), (3) of the conditions for an absolute basis cone and in addition having a compact metrizable base, then Choquet's theorem would imply that each $P_{x}$ is compact and metrizable and hence that the conclusion of Theorem 3 ' would hold. However, the conditions for an absolute basis cone do not imply Choquet's conditions. In the Banach space $l^{1}$ of real summable sequences, the elements $\left\{x_{i}\right\}=\{(0,0, \cdots$, $0,1,0, \cdots)\}$ with 1 in the $i$ th place form a basis and hence the conditions of Theorem $3^{\prime}$ hold. However, the cone $K$ of positive elements has no compact base since the existence of a compact base would imply that the hyperplane containing the basis elements would intersect $K$ in a compact set which is obviously not the case since the sequence $\left\{x_{i}\right\}$ contains no convergent subsequence. Thus Choquet's hypotheses do not hold in this case. In fact, it can be seen that Choquet's hypothesis of a compact metrizable base can never be satisfied by an absolute basis cone. Assume that $L$ is a translate of a closed hyperplane which intersects every ray of $K$ and such that $K \cap L$ is compact and metrizable. Let $x_{\alpha}=r_{\alpha} \cap L, \alpha \in A$. Then $\left\{x_{\alpha}\right\}$ is a bounded set of elements and it is possible to form a net from these elements which must contain a convergent subnet by the compactness of $K \cap L$. Assume that $\lim _{\lambda} x_{\alpha_{\lambda}}=$ $x_{0} \in L$. Hence $x_{0} \neq \theta$. However this implies that $x_{0} \in L_{\alpha}$ for every $\alpha \in A$ since if $\alpha \neq \alpha_{\lambda}$ for any $\lambda$ then $x_{\alpha_{\lambda}} \in L_{\alpha}$ for every $\lambda$ and if $\alpha=\alpha_{\lambda_{0}}$ for some $\lambda_{0}$, the net $\left\{x_{\alpha_{\lambda}}\right\}, \lambda \neq \lambda_{0}$ also converges to $x_{0}$ and $\left\{x_{\alpha_{\lambda}}\right\} \in L_{\alpha}$ for $\lambda \neq \lambda_{0}$. Thus $x_{0} \in \bigcap_{\alpha \in A} L_{\alpha}=\theta$ contrary to assumption. Hence an absolute basis cone cannot satisfy the Choquet conditions.

5. Remarks on other types of bases. If $\left\{x_{\alpha}\right\}$ is a Hamel base, the convex hull $C$ of the set of rays $r_{\alpha}=\left\{x=\lambda x_{\alpha} \mid \lambda \geqq 0\right\}$ generates all of $X$. It is easily seen that $C$ is a $C$-cone since it is the positive cone for a lattice ordering on $X$ defined by $y \geqq x$ if $y=\sum_{\alpha} a_{\alpha} x_{\alpha}, x=\sum_{\alpha} b_{\alpha} x_{\alpha}$ where the $a_{\alpha}, b_{\alpha}$ are nonzero for only finite sets of $\alpha \in A$ and if $a_{\alpha} \geqq b_{\alpha}$, $\alpha \in A$. By similar reasoning as in Theorem 3, it can be seen that necessary and sufficient conditions that $C$ be the positive cone determined by a Hamel base with the order as defined above are

(a) $C$ is the convex hull of a set of rays $\left\{r_{\alpha}\right\}, \alpha \in A$ where the linear space generated by the $\left\{r_{\alpha}\right\}$ is $X$.

(b) If $\Delta, \Gamma$ are two disjoint finite subsets of $A$ and if $L_{\Delta}, L_{T}$ are the linear subspaces generated by $\left\{r_{i}\right\}, \delta \in \Delta,\left\{r_{\gamma}\right\}, \gamma \in \Gamma$, then $L_{\Delta} \cap L_{\Gamma}=\theta$.

(c) For any $x \in C$, the set $P_{x}=C \cap(x-C)$ is finite dimensional.

A theorem of Karlin [4] shows that not all separable Banach spaces possess countable absolute bases and hence, there exist separable Banach spaces $X$ which contain no absolute basis cones with countably many 
extreme rays. In fact, Karlin's example of such a space is the space $C(a, b)$ of real valued continuous functions on a bounded closed interval. An open question which seems to be considerably more difficult than the one treated in Theorem 3 is the characterization of nonabsolute biorthogonal bases in terms of cones or of order relations. If $X$ has a nonabsolute basis $\left\{x_{\alpha}, \phi_{\alpha}\right\}$ each $x$ is uniquely representable as $x=\sum_{\alpha} \phi_{\alpha}(x) x_{\alpha}$ but the sum is no longer necessarily independent of the order of summation. It is seen as in the proof of Theorem 3 , that if $\left\{x_{\alpha}\right\}$ are elements in a nonabsolute basis and if $\left\{r_{\alpha}\right\}$ are the rays determined by the $\left\{x_{\alpha}\right\}$ and $\theta$, then the set $\left\{r_{\alpha}\right\}$ satisfies Conditions (1), (2), (3) and the set $K=\bigcap_{\alpha \in{ }_{A}} H_{\alpha}$ as defined there is a cone. However (4) is evidently not satisfied for $K$ since if it were $\left\{x_{\alpha}\right\}$ would be elements of an absolute basis. In fact, it appears unlikely than $K$ is a $C$-cone, since in the proof of Theorem 1, extensive use was made of property (4). It would be of interest to know a condition replacing (4) which would characterize $K$ as a basis cone in this case.

\section{REFERENCES}

1. G. Choquet, Existence des représentations integrales au moyen des points extremaux dans les cones convexes, C. R. Acad. Sci. Paris (1956), 699-702.

2. Unicité des représentations integrales au moyen dans les cones convex réticules, ibid., pp. 73-76.

3. R. E. Fullerton, Geometric properties of a basis in a Banach space, Proc. Int. Cong. Math., Amsterdam, 1954.

4. S. Karlin, Bases in a Banach space, Duke Math. J., 15 (1948), 971-985.

5. J. L. Kelley and R. L. Vaught, The positive cone in Banach algebras, Trans. Amer. Math. Soc., 74 (1953) 44-58.

UNIVERSITY OF MARYLAND 



\section{PACIFIC JOURNAL OF MATHEMATICS}

\section{EDITORS}

Ralph S. Phillips

Stanford University

Stanford, California

M. G. Arsove

University of Washington

Seattle 5, Washington
A. L. Whiteman

University of Southern Californla

Los Angeles 7, California

Lowell J. Paige

University of California

Los Angeles 24, California

\section{ASSOCIATE EDITORS}

E. F. BECKENBACH

D. DERRY

H. L. ROYDEN

E. G. STRAUS

T. M. CHERRY

M. OHTSUKA

E. SPANIER

F. WOLF

\section{SUPPORTING INSTITUTIONS}

UNIVERSITY OF BRITISH COLUMBIA

STANFORD UNIVERSITY

CALIFORNIA INSTITUTE OF TECHNOLOGY

UNIVERSITY OF CALIFORNIA

MONTANA STATE UNIVERSITY

UNIVERSITY OF TOKYO

UNIVERSITY OF UTAH

UNIVERSITY OF NEVADA

NEW MEXICO STATE UNIVERSITY

OREGON STATE UNIVERSITY

UNIVERSITY OF OREGON

OSAKA UNIVERSITY

WASHINGTON STATE UNIVERSITY

UNIVERSITY OF WASHINGTON

UNIVERSITY OF SOUTHERN CALIFORNIA

AMERICAN MATHEMATICAL SOCIETY CALIFORNIA RESEARCH CORPORATION SPACE TECHNOLOGY LABORATORIES NAVAL ORDNANCE TEST STATION 


\section{Pacific Journal of Mathematics}

\section{Vol. 12, No. $1 \quad$ January, 1962}

Jonathan L. Alperin, Groups with finitely many automorphisms $\ldots \ldots \ldots \ldots \ldots \ldots \ldots \ldots$

Martin Arthur Arkowitz, The generalized Whitehead product ................ 7

John D. Baum, Instability and asymptoticity in toplogical dynamics . . . . . . . . . . 25

William Aaron Beyer, Hausdorff dimension of level sets of some Rademacher series .... $\quad 35$

Frank Herbert Brownell, III, A note on Cook's wave-matrix theorem . . . . . . . . . . . . . 47

Gulbank D. Chakerian, An inequality for closed space curves ................. 53

Inge Futtrup Christensen, Some further extensions of a theorem of Marcinkiewicz ....... 59

Charles Vernon Coffman, Linear differential equations on cones in Banach spaces . . . . . 69

Eckford Cohen, Arithmetical notes. III. Certain equally distributed sets of integers . . . . . 77

John Irving Derr and Angus E. Taylor, Operators of meromorphic type with multiple poles

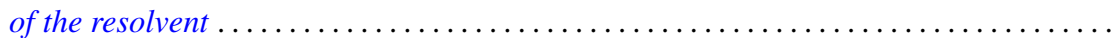

Jacob Feldman, On measurability of stochastic processes in products space .............

Robert S. Freeman, Closed extensions of the Laplace operator determined by a general class of boundary conditions, for unbounded regions ......................

Robert E. Fullerton, Geometric structure of absolute basis systems in a linear topological

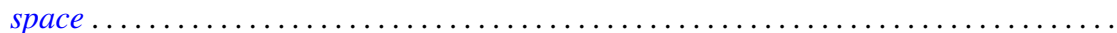

Dieter Gaier, On conformal mapping of nearly circular regions

Andrew Mattei Gleason and Hassler Whitney, The extension of linear functionals defined

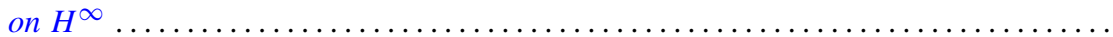

Seymour Goldberg, Closed linear operators and associated continuous linear

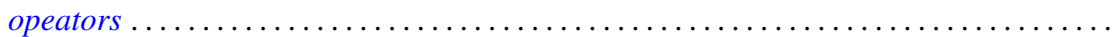

Basil Gordon, Aviezri Siegmund Fraenkel and Ernst Gabor Straus, On the determination of sets by the sets of sums of a certain order

Branko Grünbaum, The dimension of intersections of convex sets. .

Paul Daniel Hill, On the number of pure subgroups

Robert Peter Holten, Generalized Goursat problem . .

Alfred Horn, Eigenvalues of sums of Hermitian matrices ...........

Henry C. Howard, Oscillation and nonoscillation criteria for

$$
y^{\prime \prime}(x)+f(y(x)) p(x)=0
$$

Taqdir Husain, $S$-spaces and the open mapping theorem ...

Richard Eugene Isaac, Markov processes and unique stationary probability measures ...

John Rolfe Isbell, Supercomplete spaces ....................

John Rolfe Isbell, On finite-dimensional uniform spaces. II .........

N. Jacobson, A note on automorphisms of Lie algebras ..............

Antoni A. Kosinski, A theorem on families of acyclic sets and its applications

Marvin David Marcus and H. Minc, The invariance of symmetric functions of singular values...

Ralph David McWilliams, A note on weak sequential convergence.

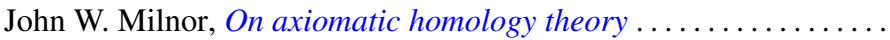

Victor Julius Mizel and Malempati Madhusudana Rao, Nonsymmetric projections in

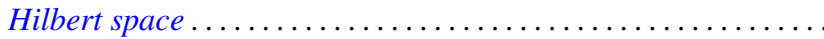

Calvin Cooper Moore, On the Frobenius reciprocity theorem for locally compact

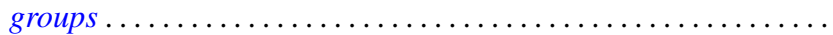

Donald J. Newman, The Gibbs phenomenon for Hausdorff means . 